\title{
Futuras alternativas para la Administración Pública
}

\section{B. Guy Peters *}

Siempre que tratamos de predecir el futuro de gobernar o de la administración pública estamos condenados, de forma casi segura, al fracaso dada la complejidad de las interacciones entre las fuerzas sociopolíticas, al igual que por la naturaleza de cambio constante de las transformaciones tecnológicas. Factores tales como la expansión de las tecnologías de la información, el creciente multiculturalismo en casi todas las sociedades y el escepticismo de los ciudadanos con respecto al gobierno, todo ello contribuye a crear un futuro muy incierto para el sector público. Este ensayo no pretende ser tanto una predicción como una constatación de las alternativas futuras para el sector público, así como un análisis de alguno de los puntos fuertes y débiles de cada una de esas alternativas.

Al presentar estas altemativas estoy argumentando de forma implícita, y en ocasiones explícitamente, a favor de una mayor consistencia en la adopción de reformas e ideas dentro del sector público que la que ha habido, ciertamente, en el pasado. Mucha de la experiencia contemporánea en la actividad gubernamental refleja una incómoda mezcla de programas y políticas deducidas de diferentes vías de pensamiento acerca del gobierno, y de diferentes diagnósticos de los problemas fundamentales con los que se enfrenta el sector público. A menudo, los programas adoptados no se integran fácilmente y pueden, de hecho, intervenir negativamente produciendo consecuencias no deseadas para la gestión de los programas públicos. Espero que de la explicación de las ideas de reformas que subyacen, y las implicaciones de esas ideas para el gobierno, pueda derivarse una consideración más atenta del futuro del gobierno $y$, junto con eso, resultar un sector público más eficiente y eficaz.

\section{Seis alternativas futuras}

Deberia enfatizar de nuevo que los siguientes seis escenarios no son tanto predicciones de futuros desarrollos, como escenarios relativamente no adulterados para posibles patrones futuros de gobiemo. No anticipo que habrá una aceptación unilineal de uno u otro de estos escenarios, en la medida que hay alguna utilidad y virtud en cada uno de ellos; más aún, la forma de gobemar actual rara vez tiene que ver con la elección de tales escenarios puros. Más bien, exponiendo este grupo de ideas debería resultar más fácil ver la naturaleza de las altemativas, cómo pueden actuar entre sí, y la variedad de opciones que los que toman las decisiones deben confrontar cuando piensan en cambiar el sector económico.

Además, la selección de uno u otro de entre esta gama de escenarios relativamente puros puede suscitar nuevos problemas para el gobiemo. Como Herbert SimON (1947) senaló hace medio siglo, la mayor parte de los consejos sobre Administración Pública tienden a darse en dicotomías, y el ir demasiado lejos a la hora de elegir un extremo u otro de la dicotomía puede producir consecuencias negativas, que, a su vez, serán la fuente de todavía otra ronda de reforma y reorganización. De tal forma, que gobernar consiste en tratar de encontrar el mejor equilibrio entre la centralización y la descentralización, organizaciones horizontales, mecanismos de control y multitud de otras variables organizacionales. De nuevo puede que no haya ninguna respuesta perfecta a alguna de estas interrogantes con respecto al gobierno, pero una comprensión más clara de estas opciones puede ayudar a tomar decisiones más adecuadas. 


\section{Continúa el statu quo (diagnóstico-agotamiento)}

La opción más lógica para el futuro de la Administración Pública es una continuación del presente y con eso la continuada incoherencia resultante de varias décadas de extensa actividad en la reforma administrativa. Al menos, desde los primeros años ochenta, ha habido un tropel de reformas en el gobierno (Peters y Savole, 1998). Esto ha sido más evidente en los sistemas políticos del tipo Westminster, pero se ha extendido virtualmente a todos los demás gobiernos. Aunque quizás dominados por el mercado como ejemplo de buena gestión (ver abajo) ha habido una amplia variedad de ideas entremezcladas en este torrente de reformas, con el resultado de que el sector público está quizás más confundido que nunca sobre su propia gestión e incluso sobre su propio sentido.

La mezcla de reformas, y de los sistemas de administración pública resultantes, refleja la variedad de ideas en juego en el cambio del sector público. Por ejemplo, por un lado, los gobiernos han estado resaltando la responsabilidad individual cara al rendimiento, al igual que gratificaciones individuales por medio de instrumentos tales como el pago de acuerdo a los resultados (EISENBERG e INGRAHAM, 1993). Esos mismos gobiemos también han estado introduciendo técnicas de gestión de tipo colectivo como el conocido "Total Quality Management" y equipos autoorganizativos en organizaciones gubernamentales (Swiss, 1992). Da la impresión de que una estructura de recompensa basada en elegir a un número relativamente pequeño de empleados para una compensación especial, probablemente no es compatible con un sistema de gestión más centrado en desarrollar y compensar la cooperación y el trabajo en grupo. En verdad, la gestión del sector privado, presumiblemente la gestión ejemplar por excelencia, tiene muchas de las mismas contradicciones internas, pero eso no hace que los problemas sean menos reales.

La razón más lógica para esperar una continuación del statu $q u o$ es el cansancio tanto político como intelectual. En primer lugar, los gobiernos ya han invertido una gran cantidad de capital político en cambiar sus estructuras internas y en elevar sus niveles de rendimiento, pero estos tipos de cambios han tendido a generar poca rentabilidad política ${ }^{1}$. Muchas de estas reformas han generado hostilidad dentro del gobiemo e indiferencia entre el público. También parece haber una escasez de nuevas ideas gerenciales que puedan incitar a los políticos o a los gestores públicos a realizar las inversiones necesarias para producir nuevas transformaciones a gran escala de la Administración Pública. Pese a que podría haber ocurrido que la cantidad de nuevos gobiernos que llegaron al poder a mediados de los noventa hubieran querido sustituir la labor de sus predecesores, parece que la reforma administrativa es sólo una prioridad para una minoría de esos gobiernos ${ }^{2}$.

Así, si la inercia domina el futuro inmediato de la Administración Pública, cabe la posibilidad de que este sistema continúe consiguiendo sus propósitos con los arreglos actuales y algo incoherentes. En verdad, debe decirse que los niveles operacionales del gobierno sí que parecen funcionar mejor que en el pasado (BoucKaERT y PoutT, 1995). Por otro lado, hay mayores aspectos de la estructura del gobierno, en su conjunto, y, de la relación entre los componentes del sector público, que no están nada claros y que pueden minimizar cualquier logro en la eficiencia a nivel operativo.

\section{La alternativa de mercado (diagnóstico-monopolio)}

Como se ha señalado arriba, la fuente dominante de ideas para la reforma del sector público en los ochenta y noventa ha sido el mercado y los modelos de los economistas. La afirmación básica que conduce mucha de esta actividad de reforma es la de que el sector público disfruta de un monopolio sobre la provisión de muchos bienes y servicios y, por tanto, no tiene ningún incentivo para volverse más eficiente (NISKANEN, 1971). Más que tratar de mejorar la eficiencia, las organizaciones gubernamentales tratan de maximizar el tamaño de sus presupuestos y de las asignaciones del personal, siendo el tamaño más que el beneficio la forma de crear rentabilidad personal para los líderes políticos y burocráticos.

Por tanto, para hacer funcionar mejor al gobierno teniendo el análisis de mercado de las dificultades en juego, la estrategia obvia es la de eliminar el monopolio y crear una competencia para los servicios gubemamentales. La competencia se puede crear a través de la privatización, de la creación de mercados intemos, o desagregando grandes departamentos en un número de agencias" cuasiautónomas (GREER, 1994; Boston et al., 1996). Incluso cuando no hay una justificación desde la teoría económica, el mercado y la competencia se asumen como las fuentes para una mejor gestión y un gobierno más eficiente. Esto es quizás particularmente cierto para la gestión del personal, con ideas tales como contratos individualizados y el pago de acuerdo al rendimiento, llegando a dominar la agenda de gestión de recursos humanos durante gran parte de las dos últimas décadas.

Muchos gobiemos, especialmente los del Reino Unido, Nueva Zelanda y Australia, ya han llevado a cabo gran parte de la agenda gerencialista de reforma basada en el modelo de mercado. En otros países, sin embargo, ha habido relativamente poco 
interés en estas ideas y casi ninguna adopción de estas reformas. Esta falta de interés es evidente incluso para otros países como Canadá, que comparte casi la misma tradición política que Gran Bretaña y Nueva Zelanda. Esto puede significar que aún queda algo de espacio para que estas reformas sean adoptadas en mayor medida, pero también puede implicar que, para algunos países, estos conceptos de gestión de base económica son incompatibles con una concepción más legalista de la vida política.

Al igual que las reformas basadas en el modelo de mercado han sido llevadas a cabo de forma diferente según los países, también estas reformas se han realizado de forma variada, según las áreas políticas de que se trate. Una de las áreas políticas más frecuentemente reformadas de acuerdo al modelo de mercado ha sido la de Sanidad (JEROME-ForGET, WHTTE y WIENER, 1995). Este patrón puede deberse, en parte, a las inmensas cantidades de dinero gastadas en atención sanitaria por los gobiernos, y también por la necesidad de ahorrar tanto de ese dinero como sea posible. El énfasis en la reforma sanitaria de acuerdo al modelo de mercado se explica, en principio, por ser éste un servicio de posible comercialización. También, otros servicios públicos han sido privatizados, en todo o en parte (MuelLer y WRIGHT, 1994). Por otro lado, pese a la variedad de propuestas y de ideas, la mayor parte de los programas de bienestar social permanecen administrados de forma muy similar a como lo eran antes del movimiento a favor del gerencialismo en el gobiemo ${ }^{3}$.

Al igual que han sido llevadas a cabo de forma diferente las reformas basadas en el modelo de mercado, también han tenido éxitos diferenciados. Por ejemplo, las reformas internas del mercado en el marco de la sanidad parecen haber tenido un mayor éxito en Suecia que en el Reino Unido, pese a la larga historia de intervención del Estado en sanidad en Suecia (JoNES, 1996). Asimismo, hay marcadas diferencias en el éxito de la privatización en diferentes áreas políticas y en diferentes países (RAMANADHAM, 1993). El mercado permanece como ejemplo poderoso para muchos críticos del business in usual en el sector público, pero no es aplicable universalmente como una idea práctica para reformar el gobierno como sus defensores quisieran creer que es.

Si hemos de pensar acerca del futuro de la Administración Pública en términos de mercado, debemos ser conocedores de los límites sustanciales de este enfoque de la actividad de gobernar. Mientras que puede que promueva una mayor eficiencia (en términos puramente económicos) dentro del gobierno, hay también muchos valores importantes que no salen bien servidos por el enfoque del modelo de mercado a la actividad de gobernar. Por ejemplo, el mercado requiere imponer una cierta variedad de responsabilidad, que no casa bien en absoluto con algunas ideas más convencionales de la responsabilidad y el deber dentro del gobierno. Igualmente, el mercado no tiene ningún mecanismo para dotar de coordinación a los poderes públicos, dado que no hay ningún mecanismo de precios efectivo en marcha en la mayoría de reformas de acuerdo al modelo de mercado en el gobiemo ${ }^{4}$. El mercado es una idea poderosa, pero también está limitada una vez que se lleva a cabo.

\section{Participación en el gobierno (diagnóstico- jerarquía)}

Mejorar la participación en las organizaciones es una alternativa a las reformas basadas en el modelo del mercado. Aunque no tan populares, o quizás tan claramente definidas, como las reformas del modelo de mercado, las reformas participativas se han llevado a cabo en varios países, y en varias áreas políticas, en las últimas décadas. Esta adopción de reformas participauvas a menudo se ha producido sin saberlo, dado que la relación de las ideas de participación no suelen estar tan a la vista como las del mercado. También, como en el mercado, el concepto de participación se utiliza para variados propósitos y es, especialmente, importante para reformar el personal y las funciones gerenciales del gobierno.

El diagnóstico obtenido de estas reformas participativas del sector público es que el gobiemo ha gestionado de una manera excesivamente jerárquica, y que los escalones más bajos de las organizaciones del gobiemo, al igual que los clientes de los programas gubernamentales, no han sido capaces de ejercer suficiente influencia sobre las decisiones adoptadas por esas organizaciones. La jerarquía ha sido uno de los elementos definitorios de las organizaciones tanto en el sector público como en el privado, y el pretender hacer las organizaciones más abiertas y democráticas apenas es una novedad. Lo que quizás sea diferente, sin embargo, es que en los noventa hay una capacidad mucho mayor para alcanzar un nivel superior de participación de forma efectiva. La mejor participación es más fácil de poner en práctica debido a que han mejorado los niveles de educación de muchos de los empleados públicos (y de sus clientes), así como la disponibilidad de tecnología para procesar la información.

La idea de la participación se ha manifestado de formas diferentes. Una de las más comunes ha sido el movimiento por la calidad en el sector público, normalmente en la forma de la -Gestión de Calidad Total. (Total Quality Management). Este es inherentemente un concepto participativo, ya que genera la implicación de los miembros de la organización en la definición 
de las metas y las operaciones de la organización. Uno de los usos de esta idea básica se encuentra en la •National Performance Review. (Gore Commission), y su promoción de ideas como las de «laboratorios de reinvención , donde se permite a las organizaciones considerar sus estructuras y procedimientos existentes y revisar esas operaciones tan extensamente como consideren necesario (PETERS y SAVOIE, 1996). Estos procesos de reinvención son de acuerdo con estándares convencionales altamente participativos y han dado lugar a importantes reestructuraciones de las organizaciones públicas. Incluso en el caso de que el gobierno no se comprometa con esfuerzos de reinvención de gran escala pueden echar abajo las jerarquías y eliminar muchos escalones de la gestión intermedia que solían controlar a los trabajadores de los escalones inferiores.

Otra manifestación del aumento en la participación en el sector público es la existencia de una mejor consulta pública sobre la política. Mientras que los gobiernos en algunos países tales como Estados Unidos han tenido una tradición de implicación pública en las decisiones políticas ${ }^{5}$, en otros ha habido un creciente énfasis en el papel del público en la políica. Por ejemplo, Canadá proviene de la tradición británica de consulta pública limitada y secreta, pero con reformas tales como el «Public Service 2000 (TeLuer, 1990) y otras reformas, se ha vuelto de forma creciente un sistema abierto al público. Asimismo, algunos países europeos han desarrollado unas nuevas vías de participación (HuNOLD, 1997).

Por último, el desarrollo de mecanismos tales como las Citizen Charter, como medio para permitir a los ciudadanos individuales obtener recompensas frente al sector público, son otra forma de participación. Mientras que estas cartas se crean principalmente como mecanismos para compensar el mal cumplimiento, o el no cumplimiento, por parte de las organizaciones del sector público, también abren nuevas vías dentro del gobierno para el público, para influir en la manera en que el gobiemo funciona. Sin duda, las "Cartas", en parte, cambian la responsabilidad del gobierno desde las instituciones representativas a otras formas más directas de responsabilidad frente al público. En cierto modo, por tanto, éste puede ser el principio de la más importante de las transformaciones del gobierno de todas las aquí discutidas.

Justo igual que las reformas basadas en el modelo de mercado, las reformas contemporáneas participativas han tenido más éxito en unos países que en otros. En especial, los conceptos participatorios han tenido más éxito en la cultura populista de los Estados Unidos y, hasta cierto punto, en la cultura canadiense, que en los escenarios políticos estatalistas de Europa, o incluso que en las Antípodas. Así como las reformas basadas en el modelo de mercado pueden ser incompatibles con sistemas en los que la ley es la fuerza primaria que define el adecuado comportamiento del gobiemo, también resulta poco probable que la partici- pación sea importante en un escenario en el que hay una tradición estatal de control centralizado sobre la política.

$\mathrm{Ha}$ habido algunas diferencias entre áreas políticas en el uso de las reformas participatorias, siendo aquellas áreas políticas con mayor número de clientes sin poder para intervenir (disempowered clients) donde más frecuentemente se localizan los cambios. Este es un conjunto políticamente interesante de cambio en el gobierno, dado que tanto a los escalones inferiores del gobierno como a sus clientes se les está diciendo simultáneamente que tienen poderes crecientes (PIERRE y PETERS, 1997). Este potencial para el conflicto por el mutuo fortalecimiento de poderes (mutual empowerment) puede potencialmente debilitar todos los resultados de las reformas participatorias del sector público. Asimismo, el fortalecimiento de poderes (empowerment) de los empleados es más factible en programas con gran número de empleados profesionales y semiprofesionales que son más capaces de participar de forma efectiva y que tiene códigos profesionales que puede modelar esa participación.

Incluso más que el mercado, el enfoque participatorio en el gobierno implica un claro y fuerte compromiso de valor. Mientras que los reformadores orientados al mercado están principalmente interesados en mejorar la eficiencia de las operaciones del gobierno, los reformistas de la participación puede que tengan metas instrumentales, pero también tienen metas más expresivas para hacer el gobierno cada vez más democrático tanto para sus trabajadores como para sus clientes. En cierta forma, estas metas democráticas son más compatibles con las metas establecidas de todos los gobiernos en el mundo de la OCDE, de lo que lo son las reformas basadas en el mercado, pero sus manifestaciones prácticas pueden desafiar la distribución de poder que prevalece dentro de muchos gobiernos y, por tanto, pueden generar algunos auténticos conflictos políticos.

En resumen, mientras que la participación es una colección de ideas algo menos coherente que dirige la reforma de lo que lo es el mercado, pese a eso ha tenido influencia en muchos escenarios nacionales. Aún más, en al menos un escenario -Estados Unidos - el enfoque de la participación ha sido el conductor dominante del cambio administrativo. Hay muchas oportunidades aún abiertas para emplear este enfoque de la actividad de gobernar con el fin de producir un cambio en el sector público, dada la concentración de la mayor parte de la gestión pública en sus propias jerarquías internas y la gestión continuada de los empleados públicos en una forma típicamente tradicional top-doun. Más aún, como el trabajo en muchas de las .funciones mecánicas" (MinTzBerG, 1979) de gobierno está siendo sustituido por tecnología informática, está disminuyendo el gran número de empleados con un nivel relativamente bajo de destreza (empleados administrativos y de secretaría en especial) que podían ser gestionados conforme a la forma autoritaria 
tradicional, y los gobiemos están teniendo que adaptar sus estilos gerenciales.

\section{El Estado flexible (diagnóstico-permanencia)}

Una de las quejas típicas con respecto al sector público es que una organización, una vez creada, es probable que permanezca durante un largo período de tiempo. Herbert Kaufman (1978), por ejemplo, argumentó que las organizaciones gubernamentales, en los EEUU, rara vez desaparecían y que gran parte del sector público está atrapado en un conjunto de organizaciones poderosas y estables. Mientras que hay una serie de interrogantes importantes acerca de la metodología que KaufMaN utilizó (PETERS y HoGwOOD, 1988), la idea de organizaciones permanentes se ha convertido en parte de la sabiduría convencional acerca del sector público.

Esta permanencia genera verdaderos problemas para quienes practican recortes presupuestarios, y también hay un gran número de otros problemas potenciales para el gobierno. En especial, estas organizaciones vinculadas a concepciones pasadas tienen que hacer frente a los problemas actuales de nuestra sociedad y a la forma en que tienen que ser tratados. A medida que cambian las tareas de gobierno, o a medida que cambia la naturaleza de los problemas públicos, al sector público le resulta difícil adaptarse debido a los compromisos que tienen con clientes y empleados de las organizaciones existentes. Más aún, la creación de estos stovepipes en el sector público hace que resulte muy dificil generar la coordinación de programas y la coherencia políica dentro del gobiemo. Estos compromisos organizacionales no son siempre, ni siquiera en los casos más frecuentes, el producto de la propia conservación simplemente, sino que muy a menudo surgen de compromisos sinceros con respecto a las metas políticas y con respecto a su clientela.

Un problema relacionado con esto es que los empleados del gobierno también son relativamente permanentes. La mayoría de los sistemas funcionariales se caracterizan por la concesión de permanencia (tenure) a sus miembros (dando por sentado su buena actuación), de tal forma que el personal en activo dificilmente se puede remover. Esta fijeza en el puesto de trabajo es operativa, aunque el mundo y sus problemas sigan adelante, y el personal empleado en el sector público no es el más apropiado para las nuevas demandas de gobierno. Esta permanencia del personal hace que la adaptación al cambio, especialmente, quizás, al cambio tecnológico, re- sulte más dificil de lo que podría ser. Eso puede hacer que el sector público sea menos eficiente de lo que de otro modo pudiera ser. De forma similar, la mayoría de los empleos públicos han sido concebidos como de jornada completa, y eso presenta problemas para las organizaciones gubernamentales que quieren ajustarse a diferentes niveles de empleo, algo que es cada vez más parte de la vida laboral en el sector privado ${ }^{6}$.

Si la permanencia es el problema para el gobierno, entonces la solución obvia es la de desarrollar los medios para reducir el grado de permanencia tanto de las organizaciones como de los contratos laborales de los individuos. Por ejemplo, de forma organizacional las opciones son desarrollar organizaciones de duración limitada (Opheim, CuRry y SHIELDS, 1994), o desarrollar organizaciones virtuales (virtual onganizations), que llegan a existir para finalidades limitadas con escasa o sin ninguna estructura formal más que un compromiso de resolver un problema en concreto (BLEECHER, 1994). Estas estructuras informales pueden involucrar a representantes de organizaciones relevantes con un compromiso para resolver el problema, y vinculadas por poco más que redes informáticas.

A nivel individual, las organizaciones gubernamentales se han vuelto más abiertas a la media jornada de trabajo y al empleo eventual. Algunas organizaciones gubernamentales (ocio y oficinas de impuestos) han utilizado esta variedad de trabajo durante algún tiempo, dado que sus operaciones se han expandido y contraído de acuerdo con la cambiante demanda. Puede ser que esta forma de empleo se esté volviendo una respuesta acorde con las exigencias de una mayor eficiencia en el gobierno. También se da un uso cada vez mayor de personal no funcionario para cubrir los puestos de gerencia superiores, especialmente para el liderazgo de organizaciones recién creadas y cuasi-independientes.

La creación de organizaciones flexibles y el uso de media jornada y de empleados no funcionarios no carece de problemas propios para el gobierno. Si las organizaciones se vuelven más efímeras, entonces, hay muchas posibilidades de que se pierda la memoria organizacional, con la posibilidad de que se repitan previos errores y de que se olviden éxitos anteriores. Asimismo, puede que los empleados públicos que no están profundamente imbuidos en los valores del servicio público no tomen el tipo de decisiones que el gobierno o los clientes querrían haber tomado. Esto es especialmente cierto si el gobierno está simultáneamente tratando de fortalecer el poder (empower) de los escalones inferiores de la burocracia para to mar más sus propias decisiones sin referencia a los status superiores en la organización. Como ocurre con todas las alternativas al statu quo en el gobiemo, hay posibles beneficios, pero también hay posibilidades reales para que se impongan costes al gobierno y a sus ciudadanos. 


\section{Gobierno desregulado (diagnóstico-demasiadas reglas)}

Otro argumento muy conocido del gobierno es que muchas de las aparentes disfunciones del sector público surgen de la existencia de demasiadas reglas —el infamante papeleo (red tape)- (KaUfman, 1977) que se supone como inherente al gobierno. Estas reglas limitan la capacidad del gobierno para responder a las condiciones cambiantes y hacen que el suministro de servicios a través del sector público resulte más caro de lo que podría ser de otro modo. Por ejemplo, la reglas de la Función Pública limitan la capacidad de los gestores públicos para contratar a quienes ellos quisieran, y seguir las reglamentaciones hace que la adquisición del equipamiento que se necesita sea lenta y pesada. Asimismo, las reglas presupuestarias existentes favorecen el exceso de gasto ${ }^{7}$ e impiden el uso flexible del dinero para proveer servicios públicos.

Una forma de ayudar a solventar los problemas del gobierno es la de eliminar tantas de esas reglas como sea posible y dejar a los gestores gestionar (let the managers manage). En Estados Unidos este movimiento para reformar el gobierno ha sido discutido como la «desregulación del gobierno" (Diluso, 1994). El National Performance Review, al igual que utilizó las ideas de participación, llevó a cabo una serie de ideas de desregulación, incluyendo materias como la eliminación de la mayoría de las regulaciones de personal puestas en vigor por la Office of Personnel Management (HORNER, 1994). En un cierto número de países las normas que regulan la compra de bienes se han relajado de tal forma que las organizaciones individuales pueden tomar sus propias decisiones. En otros países, las normas presupuestarias se han suavizado de forma que las organizaciones pueden mover dinero entre los diferentes capítulos presupuestarios y retener parte de cualquiera de los remanentes que pueden obtener, a través de una mayor eficiencia.

Como con otros enfoques a la reforma, ésta puede producir algunos beneficios pero también puede imponer costes reales al gobiemo. Las reglas que controlan el comportamiento dentro del gobierno a menudo están ahí para impedir el nepotismo, la corrupción y el favoritismo dentro de la Administración. Esas manifestaciones de corrupción son sin duda indeseables en cualquier sistema administrativo, pero quizás están también controladas tanto por los valores de un sistema de Función Pública bien institucionalizado como por medio de las numerosas e intrusivas normas. Cuando un sistema asi no se ha institucionalizado, entonces las nomas pueden ser esenciales y tratar de desregula- rizar sin los adecuados soportes valorativos puede estar condenado al fracaso.

\section{Vuelta al statu quo (diagnóstico-erosión valorativa)}

Por último, una opción para reaccionar frente al tropel de reformas de las últimas décadas es volver a algunos de los valores y los procedimientos del gobierno que existían antes de que estas reformas comenzaran. Estas reformas han producido un cierto número de resultados beneficiosos para el gobiemo y para el público, pero también han impuesto costes sustanciales. Algunos de esos costes son verdaderos costes económicos como resultado de ajustarse a un concepto cambiado de cómo debería funcionar el gobierno, pero la mayoría son más bien costes normativos. Por ejemplo, mientras que la orientación del consumidor asociada con el mercado y el gerencialismo puede llevar consigo algunos beneficios, muchos miembros del público no piensan en sí mismos como consumidores del gobierno tanto como piensan en sí mismos como ciudadanos en un sistema político en el que tienen verdaderos derechos, derechos legales. Para ellos el papel de consumidor que se les asigna por las múltiples reformas gerenciales resulta, de hecho, degradante.

Además, da igual cómo de bien preparados y de socializados estén los funcionarios, posiblemente la eliminación de muchas de las reglas por las que se han venido gobernando durante décadas pueda crear la impresión de impropiedad o incongruencia, al igual que puede abrir el camino a tal mal funcionamiento si cualquiera de los miembros del servicio público quisiera comprometerse en ello. Las reglas y las regulaciones pueden no ser ninguna garantía de que nada irá mal, pero sí que crean la imagen de que el gobierno trata de impedir ese mal funcionamiento. Más aún, esas reglas hacen el sistema de gobierno más justo para los empleados públicos. Las normas que rigen la Función Pública son, a menudo, pesadas pero tienden a dar a cualquier persona que aspire a un trabajo iguales oportunidades relativamente para acceder a él. Lo mismo cabe decir respecto de las regulaciones que controlan los mecanismos de acceso al sector público por parte de empresas privadas (obtención de contratos, por ejemplo).

Por tanto, puede tener sentido pensar acerca de una vuelta a un statu quo ante, al menos en parte. Eso, sin embargo, puede no ser tan fácil como en alguna ocasión se ha pensado que es. Mientras que muchos miembros superiores de la Función Pública se han quejado acerca de las nuevas condiciones de tra- 
bajo en las que se ven envueltos, para muchos trabajadores de escalones inferiores ha habido verdaderas mejoras en las condiciones de trabajo. Tienen más flexibilidad y más influencia real sobre lo que ellos y sus organizaciones hacen. Además, para los receptores de algunos servicios ha habido mejoras destacables en la calidad de los servicios y en la forma en que éstos son prestados. Habiendo visto las posibilidades puede resultar dificil hacer que los trabajadores o los clientes vuelvan a un sistema de gobiemo más antiguo, más jerárquico y más burocrático.

También puede resultar dificil desarrollar las coaliciones políticas necesarias para volver el sistema administrativo a sus viejas formas de prestación de servicios públicos. En primer lugar, si los servicios son de hecho mejores y los funcionarios son ahora más atentos bajo los nuevos cambios, entonces a lo mejor se encuentra menos rentabilidad política en volver al sistema anterior. Más aún, la devaluación de la Función Pública, que ha sido parte de estas reformas en muchos escenarios, ha eliminado al competidor por el poder y por la influencia sobre la política para los políticos, $y$ ha hecho posible que adopten sus propias políticas sin oposición. Esta pérdida de oposición es especialmente evidente si los funcionarios de carrera están siendo sustituidos por personal de fuera, quizás escogido con alguna influencia política. La politización del gobierno puede acentuar los virajes políticos cuando hay un cambio en el gobiemo, pero también puede dificultar la vuelta a los antiguos modos de gobierno.

\section{Resumen y conclusiones}

De nuevo debería enfatizar que este trabajo no supone un intento de predecir el futuro o de predecir la forma en que el gobierno se desarrollará durante las próximas décadas. Se trata, más bien, de entender cuáles son las opciones, y de ver cuáles son esas opciones en términos de grupos de principios relativa- mente coherentes que podrían guiar el desarrollo del sector público. Demasiado a menudo, la reforma del sector público se lleva a cabo de forma casual y desarticulada. Éste puede ser el resultado de la naturaleza del gobiemo en la mayońa de los sistemas políticos democráticos, pero no es muy probable que resulte en acuerdos de carácter político y administrativo que puedan funcionar de forma coherente y efectiva.

Es, sin embargo, mucho más fácil imponer este orden intelectual al gobiemo cuando se está sentado en Facultades o Escuelas, lejos de las batallas políicas del día a día que pueden dar forma al sector público. Cuando uno está involucrado en la refriega respecto a programas particulares, o a las presiones de intereses particulares dentro y fuera del gobiemo, puede resultar dificil resistir el adoptar reformas individuales. Más aún, el proceso de toma de decisiones en el gobierno es rara vez tan coherente, que la decisión sopesada respecto a orras y su compatibilidad, la de éstas, quede asegurada. la mayor parte de los gobiemos toman sus decisiones de forma secuencial más que como parte de un intento racional de crear una sistemática ordenación de políticas.

Lo que podemos predecir con algo de certidumbre es que el conjunto de reformas actuales del sector público no será el último. La experiencia de los profesionales del gobierno es que cada reforma engendra la siguiente ronda de reformas. La mayoría de los problemas del gobierno aparecen como grupos de dicotomías (la centralización en oposición a la descentralización, por ejemplo). Cada uno de los componentes de estas dicotomías es meritorio, pero cada uno tiene también sus riesgos. Siempre que uno de los extremos de la dicotomía se elige, esos riesgos se vuelven evidentes y puede haber una llamada a la reforma que ponga su énfasis en el otro extremo de la dicotomía. Así, la reforma vuelve a seguir a la reforma. Esto no es una reflexión de lo inadecuados que son los reformadores o de lo inapropiadas de las teorias administrativas que guían sus própositos. Más bien, es una precisa reflexión acerca de la dificultad de los problemas con los que se encuentra cualquiera que trate de gobernar una sociedad moderna.

\begin{tabular}{|c|c|c|c|c|}
\hline \multicolumn{5}{|c|}{$\begin{array}{l}\text { Tabla } 1 \\
\text { Principales rasgos de los cuatro modelos }\end{array}$} \\
\hline & $\begin{array}{l}\text { Gobierno del } \\
\text { mercado }\end{array}$ & $\begin{array}{c}\text { Gobierno } \\
\text { participativo }\end{array}$ & $\begin{array}{l}\text { Gobierno } \\
\text { flexible }\end{array}$ & $\begin{array}{c}\text { Gobierno } \\
\text { desregulado }\end{array}$ \\
\hline Principal diagnóstico & monopolio & jerarquía & permanencia & regulación interna \\
\hline Estructura & descentralización & organizaciones no jerárquicas & -organizaciones virtuales. & sin recomendación especifica \\
\hline Gestión & $\begin{array}{l}\text { pago por el funcionamientol } \\
\text { otras técnicas del sector } \\
\text { privado }\end{array}$ & $\begin{array}{l}\text { gestión de calidad total; } \\
\text { equipos }\end{array}$ & gestión de personal temporal & mayor libernad gerencial \\
\hline Adopción de decisiones & $\begin{array}{l}\text { mercados internos; } \\
\text { incentivos de mercado }\end{array}$ & consulta; negaciación & experimentación & gobiemo empresario \\
\hline Interés püblico & bajo coste & compromiso/consulta & bajo coste/coordinación & creatividad/activismo \\
\hline
\end{tabular}




\begin{tabular}{|c|c|c|c|c|}
\hline \multicolumn{5}{|c|}{ Respuestas a preguntas básicas } \\
\hline & Mercado & Participación & Flexibilidad & Desregulación \\
\hline Coordinación & -mano invisible. & de abajo arriba bottom -up & organizaciones cambiantes & interés propio de los gestores. \\
\hline $\begin{array}{l}\text { Detección y corrección } \\
\text { de errones }\end{array}$ & señales del mercado & senales políticas & $\begin{array}{l}\text { no se institucionalizan los } \\
\text { emores }\end{array}$ & aceptar más errores \\
\hline $\begin{array}{l}\text { Sistemas de la función } \\
\text { püblica }\end{array}$ & $\begin{array}{l}\text { reemplazar con mecanismos } \\
\text { de mercado }\end{array}$ & reducir la jerarquía & empleo temporal & eliminar regulaciones \\
\hline $\begin{array}{l}\text { Responsabilidad } \\
\text { (accountability) }\end{array}$ & a través del mercado & $\begin{array}{l}\text { a través de las quejas de los } \\
\text { consumidores }\end{array}$ & no hay recomendaciones claras & controles ex-pas! \\
\hline
\end{tabular}

\section{Notas}

Articulo traducido por Cristina Alba MuNoz.

- Department of Political Science. University of Pittsburgh. USA.

' Que la inversión de capital se ha dado más en términos de relaciones con sus empleados públicos que con la masa del electorado. Varias de las últimas décadas se han caracterizado por la rorura de las rutinas y los masivos ajustes llevados a cabo por los funcionarios públicos.

? El uso de workfare sí que implica uso de la labor de mercado como alternativa al soporte público, pero la lógica de la gestión intema dentro de los programas permanece poco cambiacla.

" La excepción obvia es la total privatización de las empresas que previamente eran públicas.
4 Los Estados Unidos han tenido demandas a favor de audiencias públicas para una variedad de decisiones políticas, especialmente en materia de facilidades públicas, y un número de programas sociales exigian una -máxima participación adecuada. Mientras que la efectiva participación de las clases inferiores siempre fue decepcionante, lodavía estaba ese compromiso.

' Esta faceta del empleo público está cambiando en casi todos los sistemas de función pública (ver Derlien y PEIER, 1997)

- Dado que las organizaciones no pueden retener nada del dinero que les queda en la cuenta anual de gastos, hasta el año siguiente tienen un incentivo para gastarlo, incluso si no hay ninguna razón especifica para hacerlo.

\section{Bibliografía}

BlfECKER, S. E. (1994), ·The Virtual Organization, The Futurist 28, 9-12.

Boston, J. et al. (1991), Reshaping the State (Auckland: Oxford University Press).

BouckaerT, G. y POLJTT, C. (1996), Quality Improvement in European Public Services (London: Sage).

DerLIEN, H. -U y PETERS, B. G. (1997), What Official Statistics Can Tell Us About Different Federalisms: Public Employment in Germany and the United States, paper presented to conference on -Taking the Measure of Government, Pittsburgh, PA, October 30-November 2.

Diluso, J. J. (1994), Desregulating Government (Washington, DC: The Brookings Institution.

EisenberG, E. F. e Ingraham, P. W. (1993), •Analyzing the Pay for Performance literarure: Are there Common lessons, Public Productivily and Management Review 17, $117-28$.

Greer, P. (1994), Transforming Central Govermment: The Next Steps Initiative (Buckingham: Open University Press).

HORNER, C. (1994), •Deregulating the Federal Service: I the Time Rightr, en J.J. Dilulio, ed., Deregulating the Public Service (Washington, DC: The Brookings Institution).

Hunow, C. (1997), -Deliberative Democracy and the Siting of Hazardous Facilities, Ph.D. Dissertation, Department of Political Science, University of Pittsburgh.

JONES, B. (1996), Sweden, en A. Wall, eds., Health Care Systems in Liberal Democra. cies (London: Routledge).

Kaufaran, H. (1997), Red Tape: Its Origins, Uses and Abuses (Washington, DC: The Brookings Institution).

KaufMaN, H. (1978), Are Governments Organizations Immontal? (Washington, DC: The Broookings Institution).
MintzBERG, H. (1979), The Structuring of Organizations (Englewood Cliffs, NJ: Prentice-Hall).

MUEUER, W. y WRGHT, V. (1994), :Reshaping the State in Western Europe: The Limits of Retseat, West Europeans Politics 17(3), 1-11.

NiskANEN, W. (1971), Bureaucracy and Representative Government (Chicago: Aldjne/Atherton).

OpheIm, C., CURRY, L. y ShIELDS, P. M. (1994), Sunset as Oversight, American Review of Public Admnistration 24, 253-68.

PETERS, B. G. (1996), The Future of Governing: Four Emerging Models (Lawrence, KS: University of Kansas Press).

PETers, B. G. y HoG US Federal Bureaucracy, American Review of Public Admnistration 18, 119-34.

PETERS B. G. y SAVOIE, D. J. (1998), Taking Stock: Two Decades of Administrative Reform (Montreal: McGill/Queens University Press).

Pierre, J. y Peters, B. G. (1997), CCitizens vs. The New Public Manager: The Problem of Mutual Empowerment, Unpublished paper, Department of Political Science, University of Pirsburgh.

Ramanadham, V. V. (1993), Privatization: A Global Perspective (London: Routledge).

SimoN, H. A. (1947), Administrative Behavior (New York: Free Press).

SwISS, J. (1993), •Adapting Total Quality Management (TQM) to Government, Public Administration Review 53, 356-62.

Tewuer, P. M. (1990), •Public Service 2000: The Renewal of the Public Service, Canadian Public Administration 33, 123-32. 